\title{
Algo más sobre umbrales y prototipos
}

\author{
More about thresholds and prototypes
}

Rodrigo Coloma Correa ${ }^{1}$

$Y$ te contuviste: las lágrimas no pasaron

del umbral de tus ojos.

Jorge Edwards

El Inútil de la Familia

El prototipo popular de la obstinación es la mula, animal muy inteligente.

Ambrose Bierce Diccionario del Diablo

Recepción y evaluación de propuesta: 15/04/2016

Aceptación: 15/7/2016

Recepción y aceptación final: 4/7/2017

Resumen: El texto responde a las críticas realizadas por Claudio Agüero y Raymundo Gama al texto "Los usos de los estándares de prueba: entre umbrales y prototipos", presente en esta revista.

Palabras clave: decisiones probatorias, estándares de prueba, significados de la locución estándar

1 Esta investigación es parte de proyecto financiado por el Fondo Nacional de Desarrollo Científico y Tecnológico, FONDECYT 1170872 "Prueba de los hechos. Coordinación entre el lenguaje de la teoría y el lenguaje de la práctica". 


\begin{abstract}
This article responses to the critics made by Claudio Agüero and Raymundo Gama to the essay "The uses of standards of proof: between prototypes and thresholds", content in this issue.

Keywords: evidentiary decisions, standard of proof, meanings on the notion of standard
\end{abstract}

\title{
I. Réplica a mis comentaristas
}

Los comentarios de Claudio Agüero y Raymundo Gama son agudos. La presentación de Andrés Páez, iluminadora. Todo ello constituye un acicate para transparentar lo obscuro, corregir lo erróneo y profundizar lo superficial.

Las críticas de las que me haré cargo en este espacio para réplicas se organizan en cuatro acápites. Confío resulten suficientemente representativos de lo que a mis comentaristas preocupa. Los acápites son: a) tránsito desde el lenguaje ordinario hacia el lenguaje técnico; b) uso de metáforas; c) imposibilidad de construir escalas de medición; y d) reconstrucción de la prueba/narración clara y convincente como prototipo.

\section{I.1. El tránsito desde el lenguaje ordinario hacia el lenguaje técnico (y algunas notas sobre el MARD)}

Las primeras críticas de Raymundo Gama refieren a la transferibilidad de los significados de «estándar» en el lenguaje ordinario hacia el lenguaje de los juristas. No tiene sentido negarlo. Gama tiene razón cuando sostiene que la reconstrucción de los significados atribuidos a una palabra en contextos no especializados, a veces, no aclara lo que sucede en comunidades especializadas. En consecuencia, la indagación del uso de una palabra en un contexto $A$ no siempre es útil para dar cuenta de lo que ella misma provoca en un contexto $B$.

En lo que sigue, haré precisiones acerca de por qué el uso de la partícula «estándar» en contextos extrajurídicos constituye una buena estrategia para aclarar lo que con su uso pretende comunicarse entre los juristas. El argumento principal que ofrezco en favor de que lo que ocurre en el lenguaje ordinario sirve para reconstruir significados y 
Algo más sobre umbrales y prototipos

usos en el lenguaje de los juristas descansa en la circunstancia de que la palabra "estándar» no ha adquirido en este último una fisonomía propia. Distinta es su situación en otras disciplinas, como es el caso de la estadística o de la economía. En ellas, la palabra «estándar» es utilizada con frecuencia y de una manera bastante desenvuelta. Así, si quienes aspiran a formar parte del mundo de los estadísticos o de los economistas tienen dificultades para comprender o usar la palabra «estándar» sería de esperar que consulten textos de estadística o de economía, o bien que pregunten a sus profesores o a estudiantes de cursos superiores acerca de cuál es su significado ${ }^{2}$. Buscar en los diccionarios no especializados sería insuficiente para disipar sus dudas ${ }^{3}$. Si, en cambio, quien tiene dudas acerca de lo que se quiere decir con la palabra «estándar» fuere un estudiante de derecho, encontrará un apoyo muy escaso en los textos disciplinarios, en los estudiantes más avanzados e, incluso, en sus profesores, a los efectos de aprehender significados medianamente precisos de la aludida palabra. Más le valdrá consultar un diccionario, cuestión que, por lo demás, hemos hecho distintos participantes de esta discusión para reforzar nuestros puntos de vista.

De lo recién dicho, cabría objetar que - incluso - reconociendo que sobre la palabra «estándar» ha habido una escasa labor de depuración conceptual por los juristas, pudiere ser el caso de que a lo que con su uso se apunta en contextos jurídicos diste bastante de sus significados en el lenguaje ordinario. En el fondo, es posible que cuando se usa la palabra «estándar» en la cotidianeidad se haga de una manera

2 Lo que pasa con la palabra «estándar» en dichas disciplinas podría asemejarse a lo que ocurre en el derecho cuando se intenta precisar lo que significan palabras como «regla», «principio», «nulidad» o «responsabilidad». Estas, pese a que también se ocupan con relativa frecuencia en otros contextos, en el lenguaje de los juristas tienen un nivel de depuración que incide en que el uso del diccionario provoca, en ocasiones, más confusión que claridad.

3 Lo expresado no obsta a que, de vez en cuando, sea útil tener en cuenta el significado que a esas palabras se atribuye en el lenguaje ordinario o en el de otra disciplina. A veces los tecnicismos alejan de los aspectos generales de una palabra o locución y, por ende, es útil volver sobre aquellos. La manera de hacerlo, sin embargo, será siempre desde la sospecha de que lo que ocurre en estos otros contextos pudiese no coincidir con lo que realmente interesa a la cultura de los especialistas.

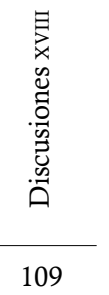


distinta a si se opera en el contexto del derecho, incluso si en este último no resulte del todo claro lo que quiere hacerse significar con ella. Para disipar esta objeción invertiré la trayectoria seguida en mi primer texto. Es decir, en vez de transitar desde el lenguaje ordinario hacia el lenguaje de los juristas, iré desde el análisis de los usos u ocurrencias de la palabra «estándar» en el mundo de los juristas hacia la noción de «estándar» en el lenguaje ordinario. Si el resultado difiriese considerablemente al de umbral y prototipo, la crítica de Gama daría en el blanco ${ }^{4}$. En caso contrario, el análisis del lenguaje ordinario conservaría sentido. Usaré como ejemplo el contexto chileno pues, además de ser el que mejor conozco, la reducida cantidad de años en que la discusión sobre $\mathrm{EdP}^{5}$ ha estado sobre el tapete, simplifica el análisis.

Si Ud. busca la palabra estándar en los códigos chilenos perderá su tiempo. Se trata de una palabra que no se usa en esos textos ${ }^{6}$. Por cierto, hay leyes especializadas en que aquello ocurre. Es el caso, por ejemplo, de la Ley General de Educación en la que se habla de «estándares de aprendizaje», «estándares de desempeño» y «estándares de calidad». El asunto es que lo que allí pudiere querer decirse con la palabra «estándar» no ilustra el significado que tiene para los juristas, pues el lenguaje que allí realmente interesa es el de los educadores ${ }^{7}$. La falta de uso de la palabra «estándar» en los textos legislados constituye un buen argumento para sostener que (parte de) la comunidad de los

4 Esta afirmación podría ser algo injusta con Gama. Lo que a él realmente le preocupa es que en mi primer texto no resultaría transparente dicha relación y, por tanto, yo debiera hacerme cargo de aquella.

5 Entre los juristas chilenos no ha habido discusión sobre el significado de «estándar».

6 Es importante hacer notar que en el proceso de tramitación del código procesal chileno (año 2000) se ha dejado constancia de que "La Comisión Mixta tuvo presente que el estándar de convicción "más allá de toda duda razonable" es propio del derecho anglosajón, y no del europeo continental, por lo que resulta una novedad también para el ordenamiento jurídico chileno [...] Historia de la Ley $N^{\circ} 19.696$ que establece Código Procesal Penal (p.2005).

7 Los intérpretes de la ley debiesen preferir prima facie el significado que dan los educadores por sobre el que le dan los juristas, si pretenden ser deferentes con una presunta intención del texto o del legislador. Si Ud. entra al sitio web: http://www.curriculumenlineamineduc.cl/ encontrará los estándares de aprendizaje de distintas asignaturas que conforman el currículum definido por el Ministerio de Educación chileno. 
Algo más sobre umbrales y prototipos

juristas es quien ha decidido que su léxico se enriquecería si se apropia de dicha palabra. La pregunta es ¿por qué?

El uso más indiscutido de la palabra «estándar» entre los juristas chilenos corresponde, precisamente, a la locución EdP. Así, los juristas cuando leen el artículo 340 del Código Procesal Penal — que habla de duda razonable pero no usa la palabra «estándar» ${ }^{8}$ - recurren a tal etiqueta bajo el entendido de que facilita la comunicación y, por cierto, su aplicación en casos concretos ${ }^{9}$. Los juristas no recurren a nomenclaturas tales como regla de la duda razonable o principio de la duda razonable, a pesar de que son frecuentes al referir a otras clases de asuntos. La negativa al uso de las palabras «principio» o «regla» — es de presumir — ocurre, en primer lugar, porque si lo hicieran se alejarían del lenguaje normal de los juristas extranjeros (quienes desde hace un buen tiempo hablan de $\mathrm{EdP}^{10}$ ) y, en segundo lugar, porque las nociones de regla y de principio, resultan incómodas para dar cuenta de lo que puede hacerse con el aludido artículo 340. Exploremos brevemente la segunda de las razones.

La comprensión del artículo 340 del Código Procesal Penal como una regla constituye un proyecto escasamente viable. Cuando se usan reglas no resulta tolerable un nivel elevado de abstracción. Aquello se explica porque el uso de las reglas no se lleva bien con el traspaso velado - ya sea a la doctrina o a los jueces - de responsabilidades que el legislador usualmente se reserva para si ${ }^{11}$; en especial de la responsabilidad de condenar o absolver en los casos de frontera.

8 En su inciso $1^{\circ}$ se dice "Nadie podrá ser condenado por delito sino cuando el tribunal que lo juzgare adquiriere, más allá de toda duda razonable, la convicción de que realmente se hubiere cometido el hecho punible objeto de la acusación y que en él hubiere correspondido al acusado una participación culpable y penada por la ley".

$9 \quad$ En Chile es el único EdP estipulado por el legislador.

10 A los juristas les interesa homologar su lenguaje y, de esa manera, no cerrar las puertas a la realización de intercambios comunicativos.

11 En la práctica, la técnica legislativa es insuficiente para inhibir la función creativa de jueces y dogmáticos lo que puede constituir una experiencia frustrante para la autoridad normativa. En el caso del EdP la frustración del legislador tal vez venga desde un carril opuesto, esto es que la doctrina y la jurisprudencia no se hagan suficientemente cargo de dotar de contenido a las respectivas disposiciones. 
La idea de operar como si se tratase de principios tampoco resulta atractiva. La abstracción del MADR no obedece a una aspiración de que cada caso requiera tener en cuenta otros principios en competición. Tampoco se debe a la circunstancia de que haya asuntos especiales de contexto de los que no se pueda desentender el intérprete en ciertos casos. La abstracción en la formulación obedece simplemente a que no se sabe cómo (o es inútil) formular, en términos más o menos precisos, los contornos entre lo que es constitutivo de duda razonable y lo que no lo es ${ }^{12}$. Sobre lo último, es útil llamar la atención en un punto insuficientemente explicitado en el texto inicial y que vale la pena tener en cuenta para los efectos de diferenciar los estándares de las reglas. Una característica relevante de los estándares es su adaptabilidad a cambios contextuales ${ }^{13}$. De un estándar se espera una rápida adaptación ante cualquier cambio tecnológico, económico, social, etc. Así, los cambios frecuentes en los estándares son vistos como inevitables e, incluso, aconsejables si se opera en entornos cambiantes. Al contrario, los cambios frecuentes en las reglas son mirados desde la sospecha.

Hay un asunto interesante en que repara Andrés Páez y que puede aclarar algo más las cosas. Dice Páez:

el concepto de estándar en el uso cotidiano asume la existencia de criterios preexistentes bien definidos a partir de los cuales se hace la medición o la comparación de similitud [...]. En contraste, en el caso de los estándares de prueba en el derecho lo que está en juego es justamente la definición de los criterios apropiados para darle contenido a dicho concepto ${ }^{14}$.

12 Es interesante tener en consideración que el aludido texto de la Historia de la ley (supra 6) continúa indicando: "Sin embargo, [el más allá de toda duda razonable] es un concepto útil, toda vez que está suficientemente decantado y elimina las discusiones relativas al grado de convicción que se requiere, dejando en evidencia que no se trata de una convicción absoluta, sino de aquella que excluya las dudas más importantes".

13 Ver 2d) en "Los usos de los estándares de prueba: entre umbrales y prototipos".

14 En rigor, no creo que lo que dice Páez dé cuenta de que los estándares en el lenguaje ordinario y en el de los juristas sean algo muy distinto. De lo que sí podría dar cuenta es que en la cotidianeidad no solemos hablar de un estándar (o de aplicar un estándar), sino a partir de que se encuentra socialmente aceptado. Los juristas hablan de estándar 
Algo más sobre umbrales y prototipos

Páez está en lo cierto, al menos cuando nos referimos a los EdP. Las fórmulas a las que recurrimos parecen vacías.

Según ha sido anticipado, hay razones para entender que la doctrina y la jurisprudencia son las responsables de dotarlas de contenido. La pregunta que cabe hacerse es - parafraseando a Laudan-: icontamos o no con un EdP mientras no se dote de contenido a sus formulaciones originarias? La respuesta debiera ser negativa (e imagino que Páez opina igual). Lo que sucede es que el estándar solo opera como tal (existe) si es posible usarlo en las distintas tareas identificadas en el texto inicial de esta discusión ${ }^{15}$.

Volvamos al parco art. 340 que dice: "Nadie podrá ser condenado por delito sino cuando el tribunal que lo juzgare adquiriere, más allá de toda duda razonable, la convicción de [...]"16. Tal como allí aparece, se trata de una disposición que requiere de una intensa labor interpretativa para hacerla operativa. Por de pronto, aquella admite reconstruirse ya sea como un permiso, un deber o una prohibición. Es habitual que se elija este último operador deóntico ${ }^{17}$. Lo que es prohibido, permitido u obligado — dependiendo de la posición que se adopte—es la acción de condenar al acusado. Del texto no es arriesgado sostener que la posición deóntica del juez — entendido como destinatario de la disposición ${ }^{18}$ - se hace depender de la adquisición de una convicción de que se ha cometido una conducta punible y que al acusado participó en ella. Lo propiamente constitutivo del estándar refiere a una propiedad predicable de la convicción; esto es su intensidad ${ }^{19} \mathrm{o}$ su clase $^{20}$.

desde el momento en que consideran existe una obligación de definirlo — mediante la interpretación - a partir de lo que diga el legislador. Es decir, poco les importa que no se haya avanzado en su depuración.

15 En tal sentido, lo que dice el legislador constituye una formulación que, es de esperar, sea utilizada como un estándar. Tal pretensión podría resultar fallida.

${ }^{16}$ Las cursivas, evidentemente, son mías.

17 Tal elección — creo- está más determinada por razones valóricas antes que técnicas.

18 También podría considerarse como destinatario al acusado si la interpretación apunta a entenderlo como una inmunidad.

19 Aquello ocurre si se reconstruye la convicción como un estado subjetivo.

${ }^{20}$ Aquello alude a perspectivas que ponen la atención en el uso de cierta metodología, en la elaboración de una cierta clase de discurso, etcétera. 
Una interpretación deferente con el texto del art. 340 (suponiendo que aquello fuere posible) debiese asumir que para condenar no es exigible la ausencia de dudas razonables, sino su neutralización. No olvidemos que se habla de "más allá de" y aquello no implica necesariamente la eliminación o ausencia de dudas más razonables. En otras palabras, se podría plantear algo así como: las dudas razonables planteadas no son de una magnitud suficiente como para inhibir una decisión que las entienda como superadas. Así, a pesar de esas dudas, estoy convencido de que/estoy autorizado a operar como si [...].

El análisis precedente resulta frustrante. Con lo dicho hasta este momento es presumible que ante un mismo caso habrá para algunos juzgadores un estado de todas-las-dudas-razonables-neutralizadas y para otros, de no-todas-las-dudas-razonables-neutralizadas. Esto constituye una invitación a dotar creativamente de contenido al texto. Si se opta por la técnica de transformar la disposición en un estándar se podrá seguir el camino ya sea de los umbrales o de los prototipos.

Apartémonos ahora del art. 340 del Código Procesal Penal. Tengamos en cuenta solo la locución «más allá de toda duda razonable», sin encorsetarla en el caso chileno. Según he enfatizado, las disposiciones del legislador solo resultan operativas en la medida en que la doctrina y la jurisprudencia realicen una importante tarea (re)constructiva de los EdP. La teoría del derecho y, más tímidamente la dogmática, algo han hecho al respecto. Así, por ejemplo, Jordi Ferrer ${ }^{21}$ realiza un ejercicio de atribución de significado al $\mathrm{MADR}^{22}$ en que se invita a prestar atención ${ }^{23}$ a la hipótesis de la culpabilidad, en cuanto explica los datos (pruebas) disponibles y predice nuevos datos, como también, a

21 Ferrer, J., "La prueba es libertad, pero no tanto: una teoría de la prueba cuasibenthamiana", en Vázquez, C. (ed.), Estándares de prueba y prueba científica. Ensayos de epistemología jurídica, Madrid: Marcial Pons, 2013, p. 36.

22 Ver la crítica a las propuestas de Ferrer y Laudan que se realiza en Allen, R., "Los estándares de prueba y los límites del análisis jurídico", en Vásquez, C. (ed.), Estándares de prueba y prueba científica. Ensayos de epistemología jurídica, Madrid: Marcial Pons, 2013, pp. 50-56.

23 Estos serían criterios relevantes de identificación de lo constitutivo de dudas razonables. Lo que no se menciona queda fuera del abanico de posibilidades de dudas razonables. Sobre criterios y estándares, ver el texto de Páez aquí publicado. 
Algo más sobre umbrales y prototipos

las hipótesis plausibles de inocencia del acusado que den cuenta de los datos generados en el proceso judicial ${ }^{24}$. A lo indicado, él propone añadir enunciados sustitutivos de la función del «más allá de» y que serían la integración coherente, la confirmación y la refutación ${ }^{25}$, respectivamente. Dicho en otras palabras, el estado del MADR se alcanza si, copulativamente: i) se logra una explicación que integre coherentemente - y en clave de culpabilidad del acusado- los datos disponibles, ii) se confirman los datos predichos por la conjetura de culpabilidad y iii) se refutan las explicaciones compatibles con la inocencia del acusado.

La reconstrucción del estado de toda-duda-razonable-neutralizada o de su complemento de no-toda-duda-razonable-neutralizada es una tarea que se aborda, también, desde los tribunales de justicia ${ }^{26}$. Aquello ocurre con algunas sentencias - por ejemplo, R v. Smith (George Joseph) [1914-1915] ${ }^{27}$ o el asesinato de Emilienne Gerard (1917) ${ }^{28}$ en que el estado de toda-duda-razonable-neutralizada se depura de una manera suficientemente precisa como para servir de referente a futuros casos. Por su parte, casos de frontera como People v. Simpson (1995), Commonwealth v. Sacco and Vanzetti (1921) o R v. Bywaters and Thompson (1922) ${ }^{29}$ dan cuenta de estados de no-toda-duda-razo-

24 Aunque Ferrer no lo dice, podría agregarse: [...] "según la hipótesis de la defensa".

25 De la exigencia de refutación se excluyen las hipótesis ad hoc.

26 En cuanto a algunas ventajas de reconstruir el EdP como prototipo considérese el siguiente fragmento que, por lo demás, es consistente con las críticas de Agüero y de las que me hago cargo en el tercero de los problemas que he anunciado: "[...] los juicios deben pasar el test de la duda razonable -esto es, jurados o jueces no deben dudar sobre la consistencia, adecuación o plausibilidad de la interpretación que incrimina a los acusados. Estos estándares normativos de juicio parecen bastante obvios cuando son vistos implícitamente como cosas que cualquiera de nosotros somos capaces de hacer sin reflexión. Cuando son examinados por sus reglas formales o la lógica, estos estándares de juicio pasan a ser más bien formidables y misteriosos". Bennett, W. L., Feldmann, M. S., Reconstructing reality in the courtroom. Justice and Judgment in American Culture, New Brunswick, Rutgers University Press, 1984, pp. 9-10.

27 Conocido también como "Las novias en la bañera".

28 Maccormick, N., "The coherence of a case and the reasonableness of doubt", The Liverpool Law Review: Spring-Autumn, 1980, pp. 45-50.

29 Un análisis minucioso puede verse en Anderson, T., Schum, D., Twining, W., Análisis de la prueba, Madrid, Marcial Pons, 2015, pp. 205-276. 
nable-neutralizada. Todos ellos hacen posible evaluaciones desde la perspectiva de sus semejanzas o diferencias, en casos futuros.

La propuesta de Ferrer previamente aludida aspira a fijar un valor mínimo de pertenencia a una categoría. Si así fuere, la comprensión de los EdP sería la propia de un umbral (y así, lo entiende él mismo) ${ }^{30}$. Por su parte, la atención en sentencias como las recién indicadas ${ }^{31}$ sirve para hacer comparaciones y, así, es más fácil resolver problemas de pertenencia en casos situados en la zona de penumbra de la categoría de los hechos probados. Nos enfrentamos, entonces, a un prototipo.

Vamos ahora brevemente a lo que implica usar estándares en ámbitos no especializados con miras a arrojar algo de luz acerca de sus significados en el uso ordinario. Para ello consideraré la toma de decisiones respecto de asuntos que presentan cierta complejidad y en los que no se recurre a estándares, aun cuando haya situaciones que linden con sus fronteras. Amos Tverski y Daniel Kahneman han planteado ideas interesantes al respecto ${ }^{32}$. Uno de sus focos apunta hacia el papel que desempeñan distintas heurísticas en nuestras elecciones. Las heurísticas constituyen vías rápidas de adopción de decisiones al saltarse varios de los pasos que serían requeridos en un análisis minucioso. Las heurísticas resultan de mucha utilidad, aun cuando en ocasiones nos conduzcan a errores que el actuar meticuloso podría haber evitado. Las heurísticas a las que los autores aludidos prestan especial atención son las de la representatividad, disponibilidad y anclaje. La primera (representatividad) consiste en tomar decisiones según la semejanza de un caso dudoso respecto a un estereotipo ${ }^{33}$ que se construye sobre la

${ }^{30}$ En lo personal, pienso que una propuesta como la de Ferrer admite también una reconstrucción como prototipo.

${ }^{31}$ Es particularmente importante cuando esos casos son incorporados en manuales o libros de texto.

${ }^{32}$ Uno de los textos más importantes al respecto es Judgment Under Uncertainty: Heuristics and Biases publicado el año 1974 en la revista Science (vol. 185). Hay versión castellana disponible: Tversky, A. y Kahneman, D., "El juicio bajo incertidumbre: heurísticas y sesgos”, en Kahneman, D., Pensar rápido, pensar despacio, Buenos Aires: Debate, 2012 (primera edición), pp. 545-567.

${ }^{33}$ Sobre estereotipos, ver Schaufer, F., Profiles, probabilities and stereotypes, Cambridge \& London: The Belknap Press of Harvard University Press, 2003. 
Algo más sobre umbrales y prototipos

categoría de cuya pertenencia se trata de dirimir. La segunda (disponibilidad) es aquella que impulsa decisiones a partir de los datos que se encuentran más fácilmente disponibles para quien decide (casos recientes o casos de alto impacto). La tercera (anclaje) es la que se produce al no hacer ajustes suficientes a los primeros datos que se han tenido en consideración y que fijan un punto de partida del razonamiento.

Quien opera bajo el influjo de una heurística, en principio, no usa estándares. Los estándares constituyen una buena coraza para evitar errores a los que las heurísticas suelen conducir ${ }^{34}$. No se crea que actuar con estándares necesariamente trae aparejado un actuar lento (¡lo que sí puede ser lento es definir el estándar!). Los estándares ya constituidos podrían conducir a una tarea equiparable a completar una check list.

Veamos un ejemplo: debemos elegir a los once jugadores que participarán en un torneo internacional de fútbol. Es posible que tengamos una inclinación espontánea a elegir a quienes tuvieron el mejor desempeño en el último partido del torneo pasado, pues se trata de la información más fresca (heurística de la disponibilidad). También es posible que fijemos distintos criterios, como lo serían el número de goles anotados, los pases correctos, las expulsiones, etcétera y luego fijemos cantidades mínimas o máximas. Con ello estaremos aplicando un estándar en el sentido de umbral. Otra posibilidad sería identificar un equipo que en el pasado haya tenido un desempeño especialmente destacado y elegir a quienes tengan características similares a los que formaban parte de dicho equipo. Con ello estaremos aplicando un estándar en su sentido de prototipo. De esta manera, cuando resolvemos problemas de la cotidianeidad mediante la construcción de estándares, lo hacemos de manera parecida a cuando operamos en el mundo de los juristas. Las opciones que en ambos casos tenemos a la vista son las de los umbrales y de los prototipos.

34 Tverski y Kahneman ofrecen un listado de doce errores a los cuales comúnmente lleva el uso de heurísticas. Tversky, A. y Kahneman, D., "El juicio bajo incertidumbre: heurísticas y sesgos", en Kahneman, D., Pensar rápido, pensar despacio, Buenos Aires, Debate, 2012 (primera edición), pp. 547-562. En rigor, la construcción de estándares no los elimina pero al obligar a una forma de análisis más guiado debiera reducirlos. 


\section{I.2. El uso de las metáforas}

Claudio Agüero pone en tela de juicio el uso de metáforas para comunicar lo que se quiere decir con la palabra «estándar»y, por cierto, con la clase más específica de los EdP. En su opinión, tanto la palabra umbral como palabra prototipo - que, a mi juicio, dan cuenta de lo que se quiere decir con la voz «estándar»— funcionan de una manera inadecuada. El uso de ambas palabras se vincularía a un espíritu cientificista, pues con ellas se evoca la noción de patrón de medida. El problema, entonces, sería algo así como se trata de hablar con términos cientificos en un campo en el que los asuntos tratados no resultan reducibles a dicho lenguaje. En términos de Agüero, sería "mejor reconocer la carencia de conceptos que permitan describir, explicar, modelar y/o reconstruir el proceso de toma de decisiones en cuya virtud, a partir de cierto(s) paso(s) o movimiento(s), se acepta como probado un hecho $\mathrm{X}$ con base en un conjunto de medios de prueba C".

El uso de metáforas, en ocasiones, esconde confusiones. Si hay que tomarse en serio a Agüero — cuestión que se encuentra fuera de discusión- se requiere revisar dicha práctica con detenimiento ${ }^{35}$. Como contrapartida, debe señalarse que las metáforas son útiles para evitar una excesiva formalización del lenguaje utilizado en un contexto, lo que no siempre constituye una ganancia ${ }^{36}$. El uso adecuado de las metáforas incide en que con menos palabras puedan decirse más cosas. Sin metá-

35 El problema que se aborda evoca de manera casi inevitable el prólogo del Tractatus: "[...] lo que en cualquier caso puede decirse, puede decirse claramente; y de lo que no se puede hablar, hay que callar la boca”. Wittgenstein, L., Tractatus logicus-philosophicus, Madrid: Tecnos, 2008 (tercera edición reimpresión), p. 103.

36 Al respecto me tomaré ciertas licencias saliéndome ligeramente del género narrativo esperable para unos comentarios en una revista como Discusiones. Citaré las siguientes palabras de Jorge Luis Borges para ilustrar acerca del exitoso uso de la metáfora en otros contextos: "Cristo es la figura más vívida de la memoria humana [...] No usó nunca argumentos; la forma natural de su pensamiento era la metáfora. Para condenar la pomposa vanidad de los funerales afirmó que los muertos enterraran a sus muertos. Para condenar la hipocresía de los fariseos dijo que eran sepulcros blanqueados". Borges, J. L., Prólogo de Evangelios Apócrifos I. Biblioteca Personal de Jorge Luis Borges, Buenos Aires: Hyspamérica, 1985, p. 9. 
foras se reducen los puentes que facilitan el contacto entre lenguajes de distintas comunidades de hablantes.

Para no desviarme más de la cuenta de las que - me imagino - son las genuinas preocupaciones de Agüero, me referiré en lo que sigue a la utilidad de hablar de umbrales y de prototipos. Es cierto que en contextos jurídicos resulta algo extraño el uso de términos como los recién señalados. Umbral es un término asociado principalmente a la arquitectura. Sin perjuicio de ello, es también utilizado sin resquemores en otros ámbitos como es el caso de la política, la historiografía o el periodismo ${ }^{37}$. En la primera, su significado resulta bastante preciso; en los segundos, se usa con mayor laxitud ${ }^{38}$. El significado que aquí interesa es el que resulta propio del segundo contexto, según han dejado de manifiesto Agüero y Gama. Así, la definición del Diccionario de la Lengua Española de la RAE que daría mejor cuenta de la palabra umbral sería la de "valor mínimo de una magnitud a partir del cual se produce un efecto determinado" 39 . El problema es que aquella definición comunica más precisión que la que, en términos generales, es posible operar en el mundo del derecho y en los otros ámbitos recién señalados. Cabe, entonces, explorar otras posibilidades como las que sugiere el Diccionario del uso del español de María Moliner cuando en la voz umbral señala: "Punto en que se está casi dentro de cierta cosa: «Eso está en los umbrales de lo prohibido»" 40 . Así las cosas, las pretensiones de cientificidad no están determinadas necesariamente por el uso de la palabra «umbral».

Con la palabra «prototipo» ocurre algo solo parcialmente similar. Antes de referirme a ello es importante hacer una aclaración. En el texto inicial el significado que se tuvo en cuenta es el de ejemplar que calza de lleno en una categoría. En lo que atañe a los EdP, el prototipo que interesa corresponde, entonces, a casos no dudosos en cuanto a

${ }^{37}$ Su uso está rodeado de un aura de cultismo o incluso, de poesía.

${ }^{38}$ Hay, por cierto, otros ámbitos en que la palabra umbral es usada con precisión. Así, ocurre, por ejemplo, en la psicofísica cuando se habla de «umbral de percepción» o de «umbral del dolor».

39 Tanto en el texto de Agüero como en el mío se alude a esta definición.

${ }^{40}$ Diccionario del uso del español de María Moliner (cuarta edición), Madrid: Gredos, 2016. 
que los hechos discutidos se han (o no) probado. El prototipo sirve para determinar - mediante la evaluación de semejanzas y diferencias- si los hechos sobre los que se discute en un caso actual deben o no darse por probados. Un prototipo puede, también, ser entendido como un modelo para generar productos similares. Para hacerme cargo de las objeciones de Agüero tendré en cuenta esta posibilidad. La palabra prototipo se encuentra estrechamente asociada al diseño de productos. En dicho ámbito se cuenta con un significado lo suficientemente preciso como para evitar equívocos respecto a lo que se entiende por tal. La palabra también se usa en otros ámbitos con mayor soltura ${ }^{41}$. El ámbito del diseño de producto, sin embargo, no es equivalente al de la arquitectura, pues resulta más transversal. Así no solo se diseñan productos en el espacio de la ingeniería, sino también en el campo de la medicina, de la agricultura, de la informática o del arte. La concepción de prototipo que se usa en ellas no se aparta de la que se tiene en cuenta para el mundo del derecho.

Cuando se habla de prototipo - a propósito de los EdP — se tiene en consideración el diseño de un producto lingüístico, esto es, la narrativa sobre hechos, ya sea que se entiendan probados o no probados. Por supuesto, el prototipo que es posible visualizar para tales efectos -y que algunas líneas atrás vinculé, a modo de ejemplo, con algunas sentencias dictadas en casos famosos- constituye un ejemplar mucho más borroso que el que se espera en la ingeniería o en la medicina. Podría decirse, entonces, que los prototipos de los que disponemos no son lo suficientemente precisos como para servir de guía eficiente a un proceso productivo, pero eso no implica que sean inútiles para la construcción de productos lingüísticos ${ }^{42}$.

${ }^{41} \mathrm{Al}$ respecto téngase en consideración uno de los significados que se le atribuye en el diccionario de María Moliner: "Ser que reúne en sí el más alto grado las características de cierto tipo de cosas y puede representarlas: «Es el prototipo del egoísta»". Diccionario del uso del español de María Moliner (cuarta edición), Gredos, Madrid, 2016.

42 Con la palabra «umbral» ocurre algo similar. En la medida en que al hablar de EdP como umbrales se identifiquen distintos criterios de evaluación e indicadores de logro que sean aplicables de manera más o menos homogénea por los jueces — cuestión de la que por cierto aun nos encontramos lejanos - los EdP pasan a ser determinantes 
Algo más sobre umbrales y prototipos

Haré una consideración final antes de cerrar este punto acerca del uso de las metáforas. Si aceptamos el carácter convencional del significado de las palabras podemos acordar que aquello que inicialmente pudo haber sido una metáfora, tiempo después podrá dejar de serlo. Aquello ocurre cuando a la comunidad interpretativa deja de importarle el significado originariamente considerado para la construcción de la metáfora y opte por asociar la palabra directamente con su nuevo significado. Hay ámbitos en los que las palabras «umbral»o «prototipo» han perdido progresivamente su significado metafórico. De esta manera, lo que en un primer momento era una metáfora, ahora podría haber dejado de serlo. Si decimos que los EdP admiten dos significados, siendo uno de ellos el de umbral del que se hace depender que una conjetura debatida en un juicio sea dada (o no) por probada, y el otro, prototipo discursivo de cuyas semejanzas y diferencias se hace depender que una conjetura debatida en un juicio sea dada (o no) por probada, lo que estamos diciendo no opera como metáfora, al menos en su sentido más fuerte ${ }^{43}$. Esto, por cierto, dependerá de la manera en que jueces, abogados, académicos y funcionarios, a fin de cuentas, usen los EdP.

\section{I.3. La imposibilidad de construir escalas de medición}

En el texto de Agüero se aborda - sin escasear en profundidad-el problema de la construcción de las escalas de medición en el ámbito científico. La razón para hacerse cargo de un asunto interesante, pero en apariencia tan lejano a lo que habitualmente preocupa a juristas y teóricos, obedece a que, en su opinión, "se aspira a contar (y a usar) estándares de prueba para construir un modelo conceptual que haga comparables los resultados obtenidos de diferentes casos tal y como si se tratara de medir su temperatura [...]".

en la construcción de productos lingüísticos. La circunstancia de que aquello todavía no ocurra no impide reconstruir, desde ya, los EdP como umbrales; aunque nos obliga a hablar con precaución.

43 Al leer la oración de Jorge Edwards en el epígrafe es fácil concluir que con ella no se aspira a abandonar la metáfora. El valor de lo que se dice está determinado en una importante medida por su sentido metafórico. 
Agüero nos previene que hay una dificultad importante en el uso de un lenguaje matematizado, incluso si se concibe como una metáfora. E1 problema - a su juicio - es que no contamos con un trabajo de conceptualización suficiente para adoptar un método de trabajo que recurra a abstracciones como las arrojadas por balanzas, termómetros o huinchas de medir. Dicho en otras palabras: antes de ponernos a medir debiésemos tener claridad acerca si nuestros conceptos son posibles de medir. Estoy de acuerdo con esta objeción, aun cuando sospecho que el universo de autores que tratan los EdP bajo la lógica propia de las escalas de medición es más reducido del que, en principio, pareciera. En lo que no estoy de acuerdo con Agüero es que concebir los estándares como umbrales constituye una manifestación de un espiritu de cientificidad que nos hace caer en la trampa de pedir a los EdP más de lo que pueden darnos. Me referiré en primer lugar al punto de acuerdo y luego, al punto de desacuerdo.

Las escalas de medición - al menos en un sentido fuerte- suponen matematizar la realidad ${ }^{44}$. La matematización de la realidad nos autoriza para dejar de lado asuntos de detalle, lo que, en algunos ámbitos de la vida, ha facilitado grandes progresos. Los números que aparecen en las escalas de medición permiten hacer comparaciones y ordenar los resultados de nuestras evaluaciones de una manera que minimiza los desacuerdos potenciales. Afirmaciones tales como " $i t i e n e s ~ 37,8^{\circ}$ de temperatura!" o "ipesas $72 \mathrm{~kg}$ !" son reconocidas como una descripción. De ellas — cuando mucho- se pone en duda si el termómetro o balanza están bien calibrados. Sin escalas de medición habría que conformarse con afirmaciones como "ite siento algo afiebrado!" o "me parece que estás un poco más gordo”, las cuales podrán ser fácilmente controvertidas y, en consecuencia, harán más difícil decidir, por ejemplo, si tomaremos un analgésico o iniciaremos una dieta.

La construcción de escalas de medición es una aspiración que asoma en distintos ámbitos de la vida (podríamos incluso estar obsesionados con saber, por ejemplo, cuánto nos quieren nuestros hijos). Conforme dice Agüero, es importante tener claridad acerca de qué es Koyré, A., Pensar la ciencia, Barcelona: Paidós, 1994, pp. 117-145. 
Algo más sobre umbrales y prototipos

lo que se va a medir (o comparar) y cuáles son los límites a los que nos vemos enfrentados. En ese sentido deben ser entendidas sus palabras en cuanto a que: "La observabilidad de una magnitud es un resultado, no un punto de inicio ni un dato autoevidente o incuestionable. Lo mismo ocurre con la mensurabilidad de una magnitud." De otra manera, sería fácil incurrir en un problema de sustitución de las preguntas que pretendíamos fuesen respondidas al momento de iniciar nuestras exploraciones sobre posibles escalas de medición ${ }^{45}$.

En los asuntos de prueba los números han sido históricamente utilizados a costa de sobresimplificar las tareas probatorias. Aquello ha ocurrido en modelos de prueba tasada en que - como se sabe- se ha contado el número de testigos y de otras pruebas ${ }^{46}$. Es interesante advertir que hubo un momento de la historia en que la experiencia dejó de ser satisfactoria y, en consecuencia, los números fueron abandonados $^{47}$. Parece de Perogrullo que la insatisfacción con el uso de los números no tuvo que ver con cuestiones aritméticas, sino con la circunstancia de que lo que ellos medían no era útil para tomar buenas decisiones. Dicho en otras palabras, se construyó una técnica, pero se carecía de una tecnología ${ }^{48}$. Para alcanzar una tecnología se requería de una ciencia que determinase aquello en lo que debía prestarse atención para dar cuenta de manera satisfactoria de la conducta humana

45 Sobre el problema de la sustitución de preguntas ver Kahneman, D., Pensar rápido, pensar despacio, Buenos Aires: Debate, 2012, pp. 132-135.

46 Ver una apretada síntesis en Ferrajoli, L., Derecho y razón (segunda edición), Madrid: Trotta, 1997, nota 28 del capítulo 3, pp. 28-29. Pese a la minuciosa regulación establecida en relación con la evaluación de las pruebas, no se redujeron todos los espacios de discrecionalidad de los jueces. Ver Tomás y Valiente, F., El derecho penal de la monarquía absoluta (siglos XVI, XVII y XVIII), Madrid: Tecnos, 1992, pp. 180-182, 199; Nobili, M., Il principio del libero convincimento del giudice, Milano: Dott. A Giuffrè, 1974, p. 113.

${ }^{47}$ Ver Cohen, L. J., "Freedom of proof", en Twining, W. y Stein, A. (eds.), Evidence and Proof, Aldershot: Darmouth, 1992, pp. 8-9.

${ }^{48}$ En otro orden de cosas dice Koyré: "La ciencia griega, decía, no creó una verdadera tecnología, porque no elaboró física. Pero, una vez más ¿por qué no lo hizo? Según todas las apariencias porque no trató de hacerlo. Y fue, sin duda, porque no creía que fuera factible". Koyré, A., "Del mundo del 'aproximadamente' al universo de la precisión”, en Koyré, A., Pensar la ciencia, Barcelona: Paidós, 1994, p. 118. 
en el pasado. Agüero reclama, entonces, que sin esa ciencia carece de sentido la búsqueda de una tecnología ${ }^{49}$.

Las investigaciones de frontera que intentan matematizar el mundo del derecho son interesantes y, en la medida de lo posible, deben ser promovidas. Lo importante es evaluar adecuadamente sus resultados. En cuanto a esto último, los resultados alcanzados hasta el momento no se han revelado como especialmente útiles y, por lo tanto, no queda si no resignarse a esperar nuevos avances. La pregunta que debemos hacernos, entonces, es ¿qué es lo que podemos hacer con los EdP si la medición de las pruebas o esquemas argumentales solo resulta posible trastornando lo que cabe esperar de un modelo probatorio que aspira a un estatus de razonabilidad?

Vamos, entonces, al punto de desacuerdo. Las escalas de medición que estarían siendo aludidas con el término «umbral» serían aquellas que hacen posible identificar un punto o, mejor dicho, un conjunto borroso de puntos. Dependiendo del lugar donde se ubiquen, los ejemplares dudosos serán considerados dentro de la categoría o fuera de ella. Es importante aclarar que los términos en que se formulan los umbrales no aspiran a niveles de depuración equivalentes al provisto por unidades de medida como los grados Celsius, para usar el ejemplo que desarrolla Agüero. Lo máximo a lo que cabe aspirar en la actualidad - para seguir con la metáfora - sería a la construcción de termómetros que aún no logran ser calibrados entre si $^{50}$.

49 Dice Agüero que para medir una variable es necesario resolver no menos de cuatro tipos de problemas: "a) Problemas cognitivos [...],b) Problemas tecnológicos [...], c) Problemas metodológicos [...] y d) Problemas de justificación [...]". Pienso que en los problemas metodológicos y de justificación contamos con avances interesantes. Mucho más atrasados estamos en los problemas cognitivos y tecnológicos. Por cierto, el atraso en los dos primeros problemas podría hacer que tengamos que replantearnos lo ya avanzado en los dos últimos cada vez que realicemos nuevos hallazgos en aquellos.

${ }^{50}$ Las escalas de medición, en ocasiones, conducen a resultados menos espectaculares que aquellos que prima facie parecieran ofrecerse. Las escalas se presentan como exactas pero la medición es problemática. Al respecto, nos dice Kuhn: "Casi siempre en la aplicación de una teoría física hay aproximación [...] y por lo tanto, no se espera que la teoría produzca resultados exactos". Kuhn, T., "La función de la medición en la física moderna", en Kuhn, T., La tensión esencial (segunda reimpresión), México D. F.: Fondo de Cultura Económica, 1993, p. 208. Con la reconstrucción de los EdP 
Algo más sobre umbrales y prototipos

¿Es, entonces, incorrecto hablar de «umbrales» para dar cuenta de lo que se intenta hacer con algunos estándares y en especial, con los EdP? Pienso que no. Imaginemos que en un juicio se discute si Fulano manifestó (o no) su consentimiento en un contrato oral de arrendamiento. Si se aplica un EdP como el de la preponderancia de la prueba y la demandante presenta tres testigos que dicen que Fulano manifestó su voluntad de obligarse al decir: «estoy de acuerdo con los términos del contrato que acaba de ser expresado en voz alta por Zutano»; a la vez que la demandada presenta un testigo que dice que Fulano no manifestó su voluntad en obligarse, pues lo que en realidad dijo fue: «no estoy de acuerdo con los términos del contrato que Zutano acaba de expresar en voz alta». Si careciéndose de otras variables se resuelve que está probada la existencia del contrato, podría decirse que el EdP ha operado como un umbral. En este caso, el análisis ha sido puramente cuantitativo. Se me objetará, con razón, que las cosas no ocurren así, pues las declaraciones de los testigos se encuentran llena de matices ${ }^{51}$. Lo relevante es que los tribunales - de alguna manera- simplifican lo que se declara en juicio en búsqueda de poder hacer comparaciones como la recién indicada. En esos casos, se evalúan declaraciones prima facie inconmensurables al igual que ocurre, por ejemplo, con los estudiantes, cuyas respuestas - salvo pruebas de alternativas o de reproducción textual - suelen ser no estrictamente comparables. Para ellos la nota de aprobación que se fija constituirá un umbral ${ }^{52}$ que al igual que en las cuestiones probatorias no se asocia a un espíritu de cientificidad.

Una aclaración final respecto a este punto. Las dificultades de comprender los EdP como umbrales cuando no contamos con escalas de

como umbrales a fin de cuentas podría no estar aspirándose a más que - utilizando un término kuhniano - una concordancia razonable con un punto que se pre-define con un cierto nivel de abstracción.

${ }^{51}$ En el ejemplo - hay que reconocerlo- los límites entre valorar la prueba y aplicar un EdP resultan borrosos.

52 Aquel umbral, para que resulte operativo, supone definir ciertos criterios y niveles de dominio que, a fin de cuentas, sirvan para decidir si el estudiante ha demostrado saber lo suficiente como para no ser reprobado. 
medición, cuestión que he tratado de sortear en las líneas precedentes, me llevan a sostener que es más fácil reconstruirlos como prototipos. ¡Nótese que Agüero no reclama que su uso esconda un eventual espíritu de cientificidad! Para realizar un juicio de semejanza no es necesario disponer de escalas de medición. Cuando hablamos de «parecerse a», «ser semejante a», rara vez esperamos que se nos precise numéricamente cuánto se parece o se asemeja una situación a otra. Nos basta con que se nos diga que «se parece bastante», o «se asemeja mucho», donde «bastante» $\mathrm{y}$ «mucho» no resultan medibles mediante aparatos que funcionen como las balanzas o como los termómetros. Para operar con prototipos basta con una técnica ${ }^{53}$.

\section{I.4. El caso de la prueba/narración clara y convincente}

Raymundo Gama reclama que la consideración de clara y convincente (CC) como un caso de prototipo padece de un problema. A su juicio, no es la narración la que debe ser clara y convincente sino la prueba rendida. Gama tiene un punto a su favor. En el Common Law se suele hablar de clear and convincing evidence. Agradezco la observación, pues hay un paso no explicitado en mi texto inicial. A continuación, daré algunas pistas de por qué me he apartado de la literalidad de la formulación del $\mathrm{CC}^{54}$.

La palabra prueba padece de varias ambigüedades. Hacerme cargo de todas ellas me desviaría del propósito de este texto. Me limitaré, entonces, a hacer unas pocas precisiones. Entre sus posibles significados, se cuenta el de prueba como soporte de un razonamiento probatorio, es decir, como la base de una cadena argumental o relato probatorio. Un cuchillo ensangrentado, un papel firmado o las palabras pronunciadas por un testigo son, entonces, pruebas. Dicho significado es importante pues daría cuenta de lo que se quiere decir con la locu-

53 Sobre diferencias entre técnica y tecnología, ver Von Wright, G. H., Ciencia y razón. Una tentativa de orientación, Valparaíso: Universidad de Valparaíso, 1995, pp. 31-32.

54 Sobre distintas formulaciones que ha recibido el EdP del CC ver Clermont, K., Standards of Decisión in Law, Durham: Carolina Academic Press, 2013, pp. 23-26. 
Algo más sobre umbrales y prototipos

ción clear and convincing evidence. Cabe preguntarse, entonces, ¿qué es lo que podría estar comunicándose al sostener que un cuchillo ensangrentado, un papel firmado o las palabras de un testigo son prueba(s) clara(s) y convincente(s)? Una posibilidad apunta a que, por ejemplo, el mensaje ${ }^{55}$ del que da cuenta la prueba es comprensible (claro) y sirve para persuadir de algo (convincente). Dicho en otras palabras, la declaración del testigo es clara si no hay dificultades en el canal de comunicación (el auditorio escucha lo mismo que pronunció el emisor) y lo que se dice es inteligible (las palabras usadas pertenecen al diccionario básico del auditorio, la gramática que se sigue no es defectuosa y hay una adecuada relación entre la dimensión locutiva e ilocutiva del lenguaje utilizado ${ }^{56}$ ). A su vez, la aludida prueba será convincente cuando, por ejemplo, su emisor — quien no se ha mostrado dubitativo ni ha incurrido en contradicciones ${ }^{57}$ - ha comunicado un mensaje útil para aceptar una cierta conjetura ${ }^{58}$.

Según lo que precede, de la prueba podría predicarse que es clara, sin necesidad de referir a lo que trata de demostrar. Lo que, en cambio, no puede decirse es que es convincente sin vincularla a lo que se quiere demostrar ${ }^{59}$. Pero hay un punto adicional: la claridad también podría referirse a las conexiones entre lo que se quiere probar y su soporte probatorio, es decir apuntaría a una trayectoria argumentativa y, en consecuencia, a un relato. Si así fuere, lo que he señalado no presentaría mayores fisuras. Se me podrá objetar que mi reconstrucción de la prueba clara y convincente no considera que el estatus de prueba depende, precisamente, de su utilidad para demostrar o refutar ciertos hechos y, por tanto, las propiedades de ser clara y convincente deben tener en cuenta

${ }^{55}$ El foco lo pongo en el caso de los testigos, pues en los restantes casos resulta especialmente evidente que los adjetivos claro y convincente no refieren al cuchillo ni al documento.

${ }^{56}$ No hay discrepancias, por ejemplo, de que lo que se está tratando de hacer es describir y no provocar emociones.

${ }^{57}$ Más que dar cuenta de que se es convincente, con ello se da cuenta de que se es creíble.

58 El Diccionario de la Lengua Española de la RAE define convincente como "Que convence". Sobre este punto ver Van Eemeren, F. y Grootendorst, R., Los actos de habla en las discusiones argumentativas, Santiago: Universidad Diego Portales, 2013, p. 164 y ss.

${ }^{59}$ Podría decirse, entonces, que la crítica de Gama funciona respecto de la propiedad de la prueba de ser clara, pero no respecto de ser convincente. 
tal relación ${ }^{60}$. Estoy de acuerdo con la objeción y creo que ella anuncia, precisamente, que lo que debe ser claro y convincente es el relato o esquema argumental propuesto para hacer explícita tal conexión.

Tomemos como ejemplo un caso célebre como el de Las novias en la bañera ${ }^{61}$. George Joseph Smith es acusado de haber matado a su cónyuge Bessie Mundy. La habría ahogado en la bañera de una casa arrendada. La defensa sostiene que ella se había ahogado durante un ataque de epilepsia. Las pruebas acerca de cómo se produjo la muerte son indirectas. Así, se había rendido prueba en orden a que George Joseph Smith estuvo casado tres veces y sus tres esposas murieron ahogadas en una bañera. A ello se sumaban otras pruebas que apuntaban, por ejemplo, a que las tres víctimas habían testado en favor de Smith y poseían propiedades o un seguro de vida, y que en los tres casos Smith había presentado como coartada que - al momento de la muerte-él estaba fuera de casa comprando pescado, huevos o tomates. Se carecía de prueba, en cambio, de que se hubiese ejercido alguna forma de violencia sobre las víctimas y, por cierto, nadie había visto ni registrado el momento en que Bessie Mundy se había ahogado.

¿Qué es determinante para que el conjunto de pruebas disponibles superen el EdP, es decir, qué hace que sean convincentes? A mi juicio, lo que lo hace posible es una teoría del caso (un relato) que atribuya significado al conjunto de las pruebas en relación con aquello que se trata de probar. En este caso el alegato de clausura del abogado de la acusación -Archibald Bodkin - cumple dicha función. En su discurso plantea que la similitud entre los tres casos hace insostenible plantear que se trató sencillamente de coincidencias o — en palabras más moder-

${ }^{60}$ En un caso importante para la comprensión del EdP del CC se preguntó al jurado: “1. Basado en prueba clara, inequívoca y convincente, ¿Frank O’Neal Addington está enfermo mentalmente? 2. Basado en prueba clara, inequívoca y convincente, Frank O'Neal Addington requiere hospitalización en un hospital psiquiátrico para su propio bienestar y protección o para la protección de otros?". Addington v. Texas, 441 U.S. 418, 422-25 (1979).

${ }^{61}$ En este caso, el EdP aplicado era el del MADR. Dado que se trata de un EdP más exigente que el CC no hay problemas en usarlo como ejemplo. 
Algo más sobre umbrales y prototipos

nas- que lo que mejor explica el conjunto de pruebas rendidas fue que George Joseph Smith planificó fríamente la muerte de sus tres esposas ${ }^{62}$.

Una última afirmación al respecto: la afirmación de que el EdP del CC es un caso de prototipo se enfrenta a la dificultad de que - al menos entre nosotros - no se dispone de una formulación canónica. Es decir, no hay un texto respecto del cual haya consenso de que el razonamiento probatorio es claro y convincente. Aquello no implica que el EdP del CC no pueda ser usado como un prototipo, sino más bien un desafío para identificar casos nítidos de superación del aludido EdP. Así, será posible ilustrar suficientemente acerca de qué relatos / teorías del caso son claras y convincentes ${ }^{63}$.

\section{Palabras de cierre}

En la base de los discursos probatorios se advierte una tensión. Aquella conduce hacia posiciones aparentemente irreconciliables. Lo que se encuentra en juego no es del todo claro y, por ello, se recurre a múltiples etiquetas. Así, se habla de binomios rivales tales como: cognoscitivismo v/s decisionismo; atomismo v/s holismo; verdad como correspondencia $\mathrm{v} / \mathrm{s}$ verdad como coherencia; o no-narrativismo v/s

${ }^{62}$ Se dice en las conclusiones del alegato de clausura: "El motivo del prisionero ha sido demostrado, la oportunidad admitida, y la exclusión del accidente, probada. Ustedes están autorizados para analizar la prueba de las otras dos muertes para ver si la muerte de la señora Mundy fue un accidente o un hecho planeado, y si fue planeado, ¿para el beneficio de quién? [...] Los tres casos son de tales características que tan numerosa cantidad de semejanzas no pueden haber ocurrido sin un diseño. En cada caso el prisionero se casó con la víctima; en cada caso el dinero en efectivo de la mujer fue sacado desde cualquier tipo de depósito bancario en el que se encontrase; en cada caso [...]". Nota: se indican once semejanzas. Anderson, T., Twining, W. y Schum, D., Análisis de la prueba. Marcial Pons, 2015, pp. 51-52. El archivo completo del juicio está en https://archive.org/stream/trialofgeorgejos $015895 \mathrm{mbp} /$ trialofgeorgejos015895mbp_djvu.txt (última visita: 7 de agosto de 2017).

63 El asunto, en todo caso, no es sorprendente pues el EdP del CC ha sido muy escasamente problematizado entre nosotros. A diferencia de lo ocurrido con el MADR los ordenamientos jurídicos del sistema del derecho continental no han estado abiertos a su incorporación como EdP. 
narrativismo. Los conflictos a los que apunta cada una de dichas parejas no son exactamente los mismos, y —más importante aún- no se reducen a cuestiones presentes solo en el mundo de los juristas ${ }^{64}$, ni se zanjan fácilmente. Tal vez, solo puedan atemperarse. A pesar de ello, pertenecen a esa clase de problemas a los que vale la pena prestar atención.

En lo que a mí respecta, no logro convencerme de que asuntos tales como el compromiso con el garantismo procesal o la finura en el tratamiento de cuestiones epistémicas se sitúe, decididamente, en un bando u otro. Más aún, creo que un cierto nivel de tensión entre los binomios aludidos le hace mucho bien al desarrollo del razonamiento probatorio ${ }^{65}$. En tal sentido, pienso que la comprensión de los EdP como umbrales ${ }^{66}$ representa un desafío y una oportunidad interesante, sobre todo a las perspectivas situadas en el primer lugar de cada binomio. Tomarse en serio la idea de que algunos EdP operan como umbrales obliga a una reflexión mucho más profunda en asuntos tales como la epistemología del testimonio o de la prueba pericial. Por su parte, la comprensión de los EdP como prototipos, también, constituye un desafío y oportunidad interesante, sobre todo a las perspectivas

${ }^{64}$ Escribía von Wright en el año 1986, a propósito de los intentos de algunos autores de escapar del modelo clásico de la física: "El pensamiento tiende a liberarse de la visión merística [atomista] y cosificante de la naturaleza y se mueve en dirección de una visión que dé mayor importancia a las totalidades, una visión holística, donde el hiato entre el sujeto y el objeto ya no divida a la realidad en dos partes estancas. No sabemos aún qué consecuencias producirá esta nueva orientación en la visión científica del mundo y en las formas de racionalidad que el pensamiento científico constituye". Von Wright, G. H., Ciencia y razón. Una tentativa de orientación, Valparaíso: Universidad de Valparaíso, 1995, pp. 84-85.

${ }^{65}$ Hayden White, siguiendo una clasificación propuesta por Stephen Pepper, clasifica a cuatro historiadores muy importantes del siglo XIX según cuál fue su aproximación a la verdad (formistas, contextualistas, mecanicistas y organicistas) en los textos por ellos construidos. Desde su perspectiva, aquello no incide en que uno haga mejor las cosas que los restantes, sino, simplemente, en que no hacen exactamente lo mismo. White, H., Metahistoria. La imaginación histórica en la Europa del siglo XIX (quinta reimpresión), México D. F.: Fondo de Cultura Económica, 2014.

66 Sobre la articulación de los EdP con las teorías de la prueba, ver Pardo, M., "Estándares de prueba y teoría de la prueba", en Vásquez, C. (ed.), Estándares de prueba y prueba científica. Ensayos de epistemología jurídica, Madrid: Marcial Pons, 2013, pp. 99-118. 
indicadas en el segundo lugar de los binomios. Hablar de prototipos obliga a explorar decididamente en asuntos tales como el análisis de la coherencia del discurso o la distinción entre lenguajes ficcionales y no ficcionales.

Restando solo unas líneas para finalizar, Ud. se preguntará: ¿que hay con el boom del que habla Agüero? ¿Qué debemos hacer para que no sea un fenómeno puramente editorial y pasajero? Los autores del boom han logrado un éxito interesante - entre otras cosas - en la instalación de algunas palabras. Una muestra de ello lo constituye - precisamenteque estemos hablando de EdP en lugar de convicción o certeza moral. El desafío a futuro es que la sustitución de conceptos que ha venido operando, no se vea reducida puramente a un cambio de etiquetas. Dicho en otras palabras, el desafío es aprovechar las posibilidades ofrecidas.

Pienso, entonces, en Martin Waldseemüller, quien fue el primero en darse cuenta de que las tierras a las que arribó Colón eran un nuevo continente, pese a que —en su caso- nunca llegó a pisarlas.

“¿Qué le permitió llegar a esa extraordinaria deducción?”, se pregunta Todorov. A su juicio, una de las claves fue su integridad intelectual, pues le impedía ajustar los datos que recibía con las ideas a priori instaladas en su época.

Tiene una idea aproximada de la circunferencia de la Tierra y de la geografía de Asia, recibe las informaciones sobre los nuevos territorios y se da cuenta de que la hipótesis asiática es imposible [...]. Solo la existencia de un océano desconocido entre América y Asia permitiría dar coherencia a esas informaciones y Waldseemüller no intenta ajustar los datos, sino que se inventa esa existencia ${ }^{67}$.

67 Todorov, T., "El descubrimiento de América", en Todorov, T., Vivir solos juntos, Barcelona, Galaxia Gutenberg, 2011, p. 55. 


\section{Bibliografía}

Anderson, T., Schum, D., y Twining, W., Análisis de la prueba, Madrid: Marcial Pons, 2015.

Bennett, W. L. y Feldmann, M. S., Reconstructing reality in the courtroom. Justice and Judgment in American Culture, New Brunswick: Rutgers University Press, 1984.

Borges, J. L., Prólogo de Evangelios Apócrifos I. Biblioteca Personal de Jorge Luis Borges, Buenos Aires: Hyspamérica, 1985.

Clermont, K., Standards of Decisión in Law, Durham: Carolina Academic Press, 2013.

Cohen, L. J., "Freedom of proof", en Twining, W. y Stein, A. (eds.), Evidence and Proof, Aldershot: Darmouth, 1992.

Van Eemeren, F., Grootendorst, R., Los actos de habla en las discusiones argumentativas, Santiago: Universidad Diego Portales, 2013.

Ferrajoli, L., Derecho y razón (segunda edición), Madrid: Trotta, 1997.

Ferrer, J., "La prueba es libertad, pero no tanto: una teoría de la prueba cuasibenthamiana", en Vázquez, C. (ed.), Estándares de prueba y prueba científica. Ensayos de epistemología jurídica, Madrid: Marcial Pons, 2013.

Koyré, A., "Del mundo del 'aproximadamente' al universo de la precisión", en Koyré, A., Pensar la ciencia, Barcelona: Paidós, 1994. Kuhn, T., "La función de la medición en la física moderna", en Kuhn, T., La tensión esencial (segunda reimpresión), México D. F.: Fondo de Cultura Económica, 1993.

Maccormick, N., "The coherence of a case and the reasonableness of doubt", The Liverpool Law Review, Spring-Autumn, 1980.

Moliner, M., Diccionario del uso del español (cuarta edición), Madrid: Gredos, 2016.

Nobili, M., Il principio del libero convincimento del giudice, Milano: Dott. A Giuffrè, 1974.

Pardo, M., "Estándares de prueba y teoría de la prueba", en Vásquez, C. (ed.), Estándares de prueba y prueba científica. Ensayos de epistemología jurídica, Madrid: Marcial Pons, 2013. 
Schauer, F., Profiles, probabilities and stereotypes, Cambridge \& London: The Belknap Press of Harvard University Press, 2003.

Todorov, T., "El descubrimiento de América", en Todorov, T., Vivir solos juntos, Barcelona: Galaxia Gutenberg, 2011.

Tomás y Valiente, F., El derecho penal de la monarquía absoluta (siglos XVI, XVII y XVIII) (segunda edición), Madrid: Tecnos, 1992.

Tversky, A. y Kahneman, D., "El juicio bajo incertidumbre: heurísticas y sesgos", en Kahneman, D., Pensar rápido, pensar despacio, Buenos Aires: Debate, 2012.

Von Wright, G. H., Ciencia y razón. Una tentativa de orientación, Valparaíso: Universidad de Valparaíso, 1995.

White, H., Metahistoria. La imaginación histórica en la Europa del siglo XIX (quinta reimpresión), México D. F.: Fondo de Cultura Económica, 2014. Wittgenstein, L., Tractatus logicus-philosophicus (tercera edición), Madrid: Tecnos, 2008. 